\title{
Fractional order calculus: historical apologia, basic concepts and some applications
}

(Cálculo de ordem fracionária: apologia histórica, conceitos básicos e algumas aplicações)

\author{
S.A. David ${ }^{\boxplus}$, J.L. Linares e E.M.J.A. Pallone \\ Departamento de Ciências Básicas, Faculdade de Zootecnia e Engenharia de Alimentos, \\ Universidade de São Paulo, SP, Brasil \\ Recebido em 22/10/2010; Aceito em 23/6/2011; Publicado em 21/11/2011
}

\begin{abstract}
Fractional order calculus (FOC) deals with integrals and derivatives of arbitrary (i.e., non-integer) order, and shares its origins with classical integral and differential calculus. However, until recently, it has been investigated mainly from a mathematical point of view. Advances in the field of fractals have revealed its subtle relationships with fractional calculus. Nonetheless, fractional calculus is generally excluded from standard courses in mathematics, partly because many mathematicians are unfamiliar with its nature and its applications. This area has emerged as a useful tool among researchers. One of the objectives of this paper is to discuss the usefulness of fractional calculus in applied sciences and engineering. In view of the increasing interest in the development of the new paradigm, another objective is to encourage the use of this mathematical idea in various scientific areas by means of a historical apologia for the development of fractional calculus.
\end{abstract}

Keywords: fractional order calculus, non-integer order systems, dynamical systems.

O cálculo de ordem fracionária (COF) lida com derivadas e integrais de ordem arbitrária (ou seja, nãointeiros) e tem as mesmas origens do clássico cálculo diferencial e integral. No entanto, até recentemente, tem sido investigado principalmente do ponto de vista matemático. Progressos nas áreas de fractais revelaram suas sutis relações com o cálculo fracionário. No entanto, o cálculo fracionário é geralmente excluído dos cursos tradicionais de Matemática, em parte porque muitos matemáticos não estão familiarizados com a sua natureza e suas aplicações. O cálculo fracionário tem se mostrado uma ferramenta útil entre os pesquisadores. Um dos objetivos deste trabalho é discutir a utilidade do cálculo fracionário nas ciências aplicadas e na engenharia. Além disso, outro propósito é incentivar o uso dessa idéia matemática como ferramenta para pesquisadores das mais diversas áreas por meio de uma apologia histórica envolvendo o desenvolvimento do cálculo fracionário.

Palavras-chave: cálculo fracionário, sistemas de ordem não-inteira, sistemas dinâmicos.

\section{Introduction}

The theory of fractional calculus dates back to the birth of the theory of differential calculus, but its inherent complexity delayed the application of its associated concepts. In fact, fractional calculus is a natural extension of classical mathematics. Since the inception of the theory of differential and integral calculus, mathematicians such as Euler and Liouville developed their ideas about the calculation of non-integer order derivatives and integrals. Perhaps the subject would be more aptly called "integration and differentiation of arbitrary order."

Despite the work that has been done in this area, the application of fractional derivatives and integrals has been infrequent until recently. However, in recent

\footnotetext{
${ }^{1}$ E-mail: sergiodavid@usp.br.

years, advances in the theory of chaos and fractals revealed relationships with fractional derivatives and integrals, leading to renewed interest in this field [1-3].

The basic aspects of the theory of fractional calculus are outlined in [4]. Insofar as it concerns the application of its concepts, we can cite research in different areas such as viscoelastic damping [5], robotics and control [6-8], signal processing [9], and electric circuits [10,11].

As for the adoption of this concept in other scientific areas, several researchers have been inspired to examine this new possibility.

Some work has been carried out in the field of dynamical systems theory, but the proposed models and algorithms are still in the preliminary stage.

With these ideas in mind, this work introduces the fundamentals of fractional order calculus (FOC) and its 
applications. This paper is organized as follows. Section 2 outlines the origins of fractional calculus, providing the background for a historical apologia for its development, and discussing several approaches to mathematical formulation. Section 3 offers examples of applications. Finally, section 4 presents a discussion, conclusions and outlook.

\section{Historical apologia and different ap- proaches}

Historically, fractional order calculus (FOC) has been unexplored or its applications delayed in engineering because of its inherent complexity, the apparent selfsufficiency of integer order calculus (IOC), and the fact that it lacks a fully acceptable geometric or physical interpretation. The intuitive idea of FOC is as old as IOC, as indicated in a letter written by Leibniz to L'Hopital in 1695. Leibniz, when asked about what if $\mathrm{n}$ were $\frac{1}{2}$ in $\frac{d^{n} y}{d x^{n}}$, said [12]: "Someday it would lead to useful consequences".

In 1730 Euler mentioned interpolating between integral orders of a derivative. In 1812 Laplace defined a fractional derivative by means of an integral, and the first discussion of a derivative of fractional order appeared in a calculus written by Lacroix in 1819 .

\subsection{Lacroix}

Lacroix expressed the $n t h$ derivative (for $n \leq m$ ) in terms of Legrende's symbol $\Gamma$ for the generalized factorial. Recalling that

$$
\Gamma(a)=\int_{0}^{\infty} t^{a-1} e^{-t} d t
$$

and starting, for instance, with the function $y=x^{m}$, Lacroix expressed it as follows

$$
\frac{d^{n} y}{d x^{n}}=\frac{m !}{(m-n) !} x^{m-n}=\frac{\Gamma(m+1)}{\Gamma(m-n+1)} x^{m-n} .
$$

Thus, replacing $n$ with $\frac{1}{2}$ and letting $m=1$, one obtains the derivative or order $\frac{1}{2}$ of the function $x$

$$
\frac{d^{1 / 2} y}{d x^{1 / 2}}=\frac{\Gamma(2)}{\Gamma(3 / 2)} x^{1 / 2}=\frac{2}{\sqrt{\pi}} \sqrt{x} .
$$

\subsection{Liouville}

It was Liouville who engaged in the first major study of fractional calculus. Liouville's first definition of a derivative of arbitrary order $\nu$ involved an infinite series. Here, the series must be convergent for some $\nu$. Liouville's second definition succeeded in giving a fractional derivative of $x^{-a}$ whenever both $\mathrm{x}$ and are positive. Based on the definite integral related to Euler's gamma integral, the integral formula can be calculated for $x^{-a}$. Note that in the integral

$$
\int_{0}^{\infty} u^{a-1} e^{-x u} d u
$$

if we change the variables $t=x u$, then

$$
\begin{aligned}
& \int_{0}^{\infty} u^{a-1} e^{-x u} d u=\int\left(\frac{t}{x}\right)^{a-1} e^{-t} \frac{1}{x} d t= \\
& \int \frac{t^{a-1}}{x^{a} x^{-1}} e^{-t} \frac{1}{x} d t=\frac{1}{x^{a}} \int_{0}^{\infty} t^{a-1} e^{-t} d t
\end{aligned}
$$

Thus,

$$
\int_{0}^{\infty} u^{a-1} e^{-x u} d u=\frac{1}{x^{a}} \int_{0}^{\infty} t^{a-1} e^{-t} d t
$$

However, in accordance with Eq. (1), this yields the integral formula

$$
x^{-a}=\frac{1}{\Gamma(a)} \int_{0}^{\infty} u^{a-1} e^{-x u} d u .
$$

Consequently, by assuming that $\frac{d^{\nu}}{d x^{\nu}}\left(e^{a x}\right)=a^{\nu} e^{a x}$ for any $\nu>0$, then

$$
\begin{aligned}
& \frac{d^{\nu} x^{-a}}{d x^{\nu}}=\frac{\Gamma(a+\nu)}{\Gamma(a)} x^{-a-\nu}= \\
& (-1)^{\nu} \frac{\Gamma(a+\nu)}{\Gamma(a)} x^{-a-\nu} .
\end{aligned}
$$

The $(-1)^{\nu}$ term in the latter equation suggests the need to expand the theory to include complex numbers.

Indeed, in terms of contemporary definitions, the modern theory of fractional calculus is intimately connected with the theory of operators. In classical calculus, the symbol $D_{x}^{n}$ is often used for the $n t h$ derivative operator (for $n \geq 0$ ) while, less commonly, $D_{x}^{-1}$ is used for the anti-derivative (or integral) operator.

A convenient notation described by Davis [12] was the following: if $\nu$ is a positive real number, ${ }_{c} D_{x}^{\nu} f(x)$ denotes differentiation of order $v$ of the function $f$ along the $x$-axis. Similarly, the operator ${ }_{c} D_{x}^{-\nu} f(x)$ will denote integration of order $v$ of the function $f$ along the $x$-axis.

Fractional calculus still lacks a geometric interpretation of integration or differentiation of arbitrary order. Hence, the subscripts $c$ and $x$ are called here terminals of integration instead of limits of integration. This avoids unnecessary confusion. 


\subsection{Laurent}

In 1884 Laurent published what is now recognized as the definitive paper on the fundaments of fractional calculus. Using Cauchy's integral formula for complex valued analytical functions and a simple change of notation to employ a positive $\nu$ rather than a negative $\nu$ will now yield Laurent's definition of integration of arbitrary order $\nu>0$

$$
{ }_{c} D_{x}^{-\nu} f(x)=\frac{1}{\Gamma(\nu)}=\int_{c}^{x}(x-t)^{\nu-1} f(t) d t .
$$

The appropriate definition of differentiation of arbitrary order is to integrate it up to a point from which the desired result can be obtained by conventional differentiation.

Let $n u=m-r h o$ where, for convenience, $\mathrm{m}$ is considered the smallest integer larger than $\nu$ and $0<\rho \leq 1$.

Observe that

$$
{ }_{c} D_{x}^{\nu} f(x)={ }_{c} D_{x}^{m-\rho} f(x)
$$

Thus

$$
{ }_{c} D_{x}^{m-\rho} f(x)=\frac{d^{m}}{d x^{m}}\left[{ }_{c} D_{x}^{-\rho} f(x)\right],
$$

and consequently

$$
\frac{d^{m}}{d x^{m}}\left[{ }_{c} D_{x}^{-\rho} f(x)\right]=\frac{d^{m}}{d x^{m}}\left[\frac{1}{\Gamma(\rho)} \int_{c}^{x}(x-t)^{\rho-1} f(t) d t\right] .
$$

\subsection{Cauchy}

Cauchy's definition, which is recognized as one that preserves some important frequency properties [13], is expressed as follows

$$
f_{+}^{(\alpha)}=\int f(\tau) \frac{(t-\tau)^{-\alpha-1}}{\Gamma(-\alpha)} d \tau,
$$

where the function $\Gamma(\alpha)$ is the generalization of factorial function and is defined in Eq. (1).

Indeed, since the birth of differential and integral calculus, the generalization of the derivative and integral concept to a non-integer order has been the subject of several approaches. For this reason, there are various definitions that are provenly equivalent, and their use can (and should) be encouraged by researchers in different scientific areas.

\subsection{Caputo}

In order to present Caputo's fractional derivative, let $m$ be the smallest integer that exceeds $\alpha$, thus enabling Caputo's fractional derivative of order $\alpha>0$ to be defined as follows

$$
\begin{aligned}
& D_{*}^{\alpha} f(t)=\frac{1}{\Gamma(m-\alpha)} \int_{0}^{t} \frac{f^{(m)}(\tau)}{(t-\tau)^{\alpha+1-m}} d \tau, \\
& m-1<\alpha<m .
\end{aligned}
$$

Clearly, numerous mathematicians have contributed to the history of fractional calculus by attempting to solve a fundamental problem to the best of their understanding.

Each researcher sought a definition and therefore different approaches, which has led to various definitions of differentiation and antidifferentiation of noninteger orders that are provenly equivalent. Some of the aforementioned definitions of non-integer order derivatives are summarized and listed in Table 1. A table can also be written for the definition of different and equivalent integrals.

Although all these definitions may be equivalent, from one specific standpoint, i.e., for a specific application, some definitions seem more attractive.

Table 1 - Definition of derivatives of arbitrary order.

\begin{tabular}{l}
\hline $\begin{array}{l}\frac{d^{n} y}{d x^{n}} \\
\text { Lacroix }\end{array}$ \\
$\qquad \frac{d^{\nu} x^{-a}}{d x^{\nu}}=(-1)^{\nu} \frac{\Gamma(m+1)}{\Gamma(a)} x^{-a-\nu}$ \\
Liouville \\
Laurent $\quad{ }_{c} D_{x}^{\nu} f(x)={ }_{c} D_{x}^{m-\rho} f(x)=\frac{d^{m}}{d x^{m}}\left[\frac{1}{\Gamma(\rho)} \int_{c}^{x}(x-t)^{\rho-1} f(t) d t\right]$ \\
Cauchy $\quad f_{+}^{(\alpha)}=\int f(\tau) \frac{(t-\tau)^{-\alpha-1}}{\Gamma(-\alpha)} d \tau$ \\
Caputo $\quad D_{*}^{\alpha} f(t)=\frac{1}{\Gamma(m-\alpha)} \int_{0}^{t} \frac{f^{(m)}(\tau)}{(t-\tau)^{\alpha+1-m}} d \tau$
\end{tabular}




\section{Applications in applied sciences and engineering}

Fractional order calculus can represent systems with high-order dynamics and complex nonlinear phenomena using few coefficients, since the arbitrary order of the derivatives provides an additional degree of freedom to fit a specific behavior. Another important characteristic is that fractional order derivatives depend not only on local conditions of the evaluated time but also on the entire history of the function. This fact is often useful when the system has a long-term "memory" and any evaluation point depends on the past values of the function.

At this point we therefore consider it relevant to present some applications involving the implementation of FOC-based models in different physical systems, namely: the diffusion equation, food engineering, robotics and control theory, and econophysics.

\subsection{Applications in the diffusion equation}

It is well known that, in the classical case, the diffusion equation is given by

$$
\frac{\partial u}{\partial t}=b^{2} \frac{\partial^{2} u}{\partial x^{2}}
$$

A general solution is given for a fractional diffusion equation defined in a bounded space domain. The fractional time derivative is described in the Caputo sense (Table 1). The Caputo fractional derivative is considered here because it allows for the standard inclusion of traditional initial and boundary conditions in the formulation, whereas models based on other fractional derivatives may require the values of the fractional derivative terms at the initial time. Keeping this definition in mind, the fractional diffusion equation [14] of interest can be written as

$$
\frac{\partial^{\alpha} u}{\partial t^{\alpha}}=b^{2} \frac{\partial^{2} u}{\partial x^{2}}
$$

where $\alpha$ is a parameter describing the order of the fractional derivative, $b$ denotes a constant coefficient with dimension (Time) $(\text { Length })^{-\alpha / 2}, x$ and $t$ are the space and time variables, and $u=u(x, t)$ is the field defined in the space domain $[0, L]$, considering the following boundary conditions

$$
\begin{aligned}
& u(0, t)=u(L, t)=0, \quad t>0, \\
& u(x, 0)=f(x), \quad 0<x<L, \\
& u_{t}(x, 0)=0, \quad 0<x<L, \\
& \text { for } 1<\alpha \leq 2 .
\end{aligned}
$$

The last boundary condition is assumed to ensure the continuous dependence of the solution on the parameter $\alpha$ in the transition from $\alpha=1^{-}$to $\alpha=1^{+}[2]$.
Taking the finite sine transform of Eq. (16), integrating the second term of the resulting equation by parts, and applying the boundary conditions, we obtain

$$
\frac{d^{\alpha} \bar{u}}{d t^{\alpha}}+(\text { ban })^{2} \bar{u}=0
$$

and

$$
\bar{u}=\bar{u}(n, t)=\int_{0}^{L} u(x, t) \sin (\operatorname{anx}) d x,
$$

is the finite sine transform of $u(x, t)$, where $a=\pi / L$, and $\mathrm{n}$ is a wave number, Eq. (18) may be called a diffusion-wave equation in a wave number domain. Taking the finite sine transform of Eq. (17), we obtain

$$
\bar{u}(n, 0)=\int_{0}^{L} f(x) \sin (\text { anx }) d x,
$$

and $\bar{u}_{t}(n, 0)=0$ for $1<\alpha \leq 2$.

Taking the Laplace transform of Eq. (16) and using the initial conditions and the properties of the Caputo derivative, we obtain

$$
U(n, s)=\frac{s^{\alpha-1} \overline{u(n, 0)}}{s^{\alpha}+(b a n)^{2}}
$$

where $s$ is the Laplace transform parameter, and $U(n, s)$ is the Laplace transform of $\bar{u}(n, t)$.

Taking first the inverse Laplace transform of Eq. (21) and then the inverse finite sine transform of the resulting equation, we obtain

$$
\begin{aligned}
& u(x, t)=\frac{2}{L} \sum_{n=1}^{\infty} E_{\alpha}\left(-b^{2} a^{2} n^{2} t^{\alpha}\right) \sin (a n x) \times \\
& \int_{0}^{L} f(r) \sin (a n r) d r,
\end{aligned}
$$

where $E_{\alpha}$ is the Mittag-Leffler function.

The Mittag-Leffler function has several interesting properties.

In particular, we have $E_{1}(-z)=e^{-z}$ and $E_{2}\left(-z^{2}\right)=\cos (z)$. Using these identities, the solutions of Eq. (16) for $\alpha=1$ and 2 are given as

$$
\begin{aligned}
& u(x, t)=\frac{2}{L} \sum_{n=1}^{\infty} e^{-(\text {ban })^{2} t} \sin (\text { an } x) \times \\
& \int_{0}^{L} f(r) \sin (\text { anr }) d r
\end{aligned}
$$

and

$$
\begin{aligned}
& u(x, t)=\frac{2}{L} \sum_{n=1}^{\infty} \cos (\text { ban } t) \sin (\text { anx }) \times \\
& \int_{0}^{L} f(r) \sin (\text { anr }) d r .
\end{aligned}
$$


Equations (23) and (24) represent the diffusion and the wave solutions. These are special cases of the solution (Eq. (22)) of the fractional diffusion-wave equation.

\subsection{Applications in food engineering}

Food gums are complex carbohydrates and can serve a wide variety of functions, ranging from stabilizers to fat replacers. In many foods, these ingredients are also useful for building and/or modifying the product's texture.

In the special case where the material and thickness are uniform throughout, there will be bending stresses that can be obtained from the normal relationship

$$
\tau(t)=k \frac{d^{2} \gamma}{d t^{2}}
$$

For a material that is neither a Hookean solid nor a Newtonian fluid, Ma and Barbosa-Cánovas [15] analyzed experimental results from elastoviscous bodies obtained by Bosworth [16] and proposed the following relationship

$$
\tau(t)=k \frac{d^{\alpha} \gamma}{d t^{\alpha}}
$$

with $k=$ constant, $0 \leq \alpha \leq 1, \tau$ represents shear stress and $\gamma$ is the shear strain.

Applying the Boltzmann superposition principle in combination with the fractional derivative concept, Eq. (26) has can be written as follows

$$
\tau(t)=\sum_{n=1}^{N} K_{n} \frac{d^{\alpha_{n}} \gamma}{d t^{\alpha_{n}}} .
$$

Equation (27) rewritten in terms of a fractional operator (Laurent sense, Table 1 ) is

$$
\tau(t)=\sum_{n=1}^{N} K_{n} D^{\alpha_{n}[\gamma(t)]} .
$$

Equation (28) is an expression for the linear viscoelasticity of materials in terms of a fractional derivative. From a practical point of view, the material functions, such as dynamic viscosity $\left(\eta^{\prime}\right)$ and complex viscosity $\left(\eta^{\prime \prime}\right)$, need to be derived implicitly from stressstrain relations. Therefore, the theory of fractional derivatives, when employed to manipulate these material functions based on stress-strain relations, results in

$$
\begin{aligned}
\eta^{\prime} & =k_{1} \omega^{\alpha_{1}-1} \sin \left(\frac{\pi}{2} \alpha_{1}\right)+k_{2} \omega^{\alpha_{2}-1} \sin \left(\frac{\pi}{2} \alpha_{2}\right), \\
\eta^{\prime \prime} & =k_{1} \omega^{\alpha_{1}-1} \cos \left(\frac{\pi}{2} \alpha_{1}\right)+k_{2} \omega^{\alpha_{2}-1} \cos \left(\frac{\pi}{2} \alpha_{2}\right) .
\end{aligned}
$$

Equations (29) and (30) can then be used to simulate the linear viscoelasticity of food gums, once the constants in the equations have been determined from the experimental data.

\subsection{Applications in robotics and control the- ory}

In industrial environments, robots have to execute their tasks quickly and precisely, minimizing production time. This requires flexible robots working in large workspaces, which means that they are influenced by nonlinear and fractional order dynamic effects.

Ferreira et al.[17] analyzed the effect of a hybrid force and position fractional controller applied to two robotic arms holding the same object, as illustrated in Fig. 1.

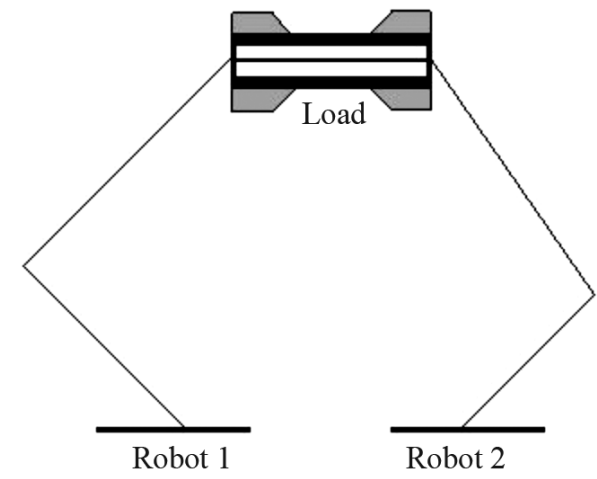

Figure 1 - A cooperative cell of robots performing a given task.

The load of the object varied and some disturbances were applied as references of force and position. Instead of using the classic PID (Proportional-IntegralDerivative) controller, they used a $\mathrm{PI}^{\alpha} \mathrm{D}^{\mu}$ controller which was calibrated by trial and error. The resulting controller proved to be robust in handling variable loads and small disturbances at the reference.

Another interesting problem in robotics which can be treated with FOC is the control of flexible robots, as this kind of light robot uses low-power actuators, with no self-destruction effects in response to high impacts. Nevertheless, significant vibrations over flexible links make a position control difficult to design, because it reveals a complex behavior that is difficult to approximate by linear differential equations [18]. However, Monje et al. [19] propose a $P D^{\alpha}$ for a flexible robot with one degree of freedom for variable loads, resulting in a system with static phase and constant overshoot, independent of the applied load.

An application in a robot with legs was presented by Silva and Machado [20], who wrote a set of $P D^{\alpha}$ algorithms to control position and force, which they applied to a hexapod robot with 12 degrees of freedom. The authors defined two performance metrics, one for quantity of energy and the other for position error. The controllers with $\alpha=0.5$ showed the best performance in this robot. 


\subsection{Applications in econophysics}

Econophysics is a new interdisciplinary area in which concepts and analysis techniques commonly used for describing physical systems are applied to investigate financial and economic problems.

The dynamics of global markets require full time complete and highly accurate modeling.

Although numerous studies about financial markets have been published [21-27], only a few of them use fractional calculus as a tool. Nevertheless, some investigations in finance using fractional calculus equations were made by Scalas et al. [28].

David [29] proposed a very simple model based on the following discussion: It is well- known that a significant change in capital flow commonly occurs when investors, at any moment, perceive a minor change in risk.

It seems to me reasonable to imagine that the square of the capital flow invested, denoted as $\left(\frac{d \lambda}{d t}\right)^{2}$, can be proportional to the dimensionless perception of this risk variation, denoted as $\left(y-y_{0}\right)$.

Mathematically,

$$
\left(\frac{d \lambda}{d t}\right)^{2} \propto\left(y-y_{0}\right),
$$

can be written as

$$
\left(\frac{d \lambda}{d t}\right)^{2}=-C\left(y-y_{0}\right) .
$$

An increase in risk perception stimulates a reduction in capital injection. That is the meaning of the minus signal above. Thus

$$
\frac{d \lambda}{d t}=C^{1 / 2}\left(y_{0}-y\right)^{1 / 2},
$$

and

$$
\frac{d \lambda}{\left(y_{0}-y\right)^{1 / 2}}=C^{1 / 2} d t .
$$

Now, integrating both sides,

$$
\sqrt{C} \int_{0}^{T} d t=\int_{0}^{y_{0}}\left(y_{0}-y\right)^{-1 / 2} d \lambda,
$$

or

$$
K=\int_{0}^{y_{0}}\left(y_{0}-y\right)^{-1 / 2} d \lambda,
$$

where $K=C^{1 / 2} T$.

Here, $\lambda=F(y)$, where $\lambda$ is the amount of return on capital and $y$ represents the risk perception.

Bearing in mind this fact, we can note that $d \lambda=$ $F^{\prime}(y) d y$.
If we change variables $y_{0}$ and $y$ to $x$ and $t$, and replace $F^{\prime}$ with $f$, the integral equation becomes

$$
K=\int_{0}^{x}(x-t)^{-1 / 2} f(t) d t .
$$

From this point on the problem is to determine the function $f$. This can be done by multiplying the last equation by $1 / \Gamma(1 / 2)$ in order to obtain

$$
\begin{aligned}
& \frac{K}{\Gamma(1 / 2)}=\frac{1}{\Gamma(1 / 2)} \int_{0}^{x}(x-t)^{-1 / 2} f(t) d t= \\
& { }_{0} D_{x}^{-1 / 2} f(x) .
\end{aligned}
$$

Consequently,

$$
{ }_{0} D_{x}^{1 / 2} K=\sqrt{\pi} f(x) .
$$

Based on Laurent's general definition (Table 1) of derivatives, let us now consider the derivative of order $1 / 2$ of the constant $K$. Using $D^{\nu}=D^{m-\rho}$ we find that

$$
\begin{aligned}
& { }_{0} D_{x}^{\nu} K=\frac{d^{m}}{d x^{m}}\left[\frac{1}{\Gamma(\rho)} \int_{0}^{x}(x-t)^{\rho-1} K d t\right]= \\
& \frac{d^{m}}{d x^{m}}\left[\frac{K}{\Gamma(\rho)} \frac{x^{\rho}}{\rho}\right]=\frac{K p ! x^{\rho-m}}{(\rho-m) ! \rho \Gamma(\rho)}= \\
& \frac{K}{\Gamma(1-\nu)} x^{-\nu} .
\end{aligned}
$$

In particular, when $\nu=1 / 2$, we have

$$
{ }_{0} D_{x}^{1 / 2} K=\frac{K}{\Gamma(1 / 2)} x^{-1 / 2}=\frac{K}{\sqrt{\pi}} x^{-1 / 2} .
$$

Therefore, from Eqs. (39) and (41) it can be concluded that

$$
K=\pi \sqrt{x} f(x) .
$$

This model involving risk and capital return based on the non-integer order calculus concepts is simple to implement and offers an interesting alternative way for investigation and possibly for predictions in financial markets.

\section{Discussion and conclusions}

Fractional Order Calculus (FOC) dates back to the birth of the theory of differential calculus or Integer Order Calculus (IOC). However, FOC only began to be applied in the last two decades as a result of advances in the area of chaos, which revealed subtle relationships with the FOC concepts. 
Recent progress in the area of fractional derivatives and integrals implies a promising potential for future developments and application of the theory in various scientific areas. Some basic concepts of FOC and several applications in applied sciences and engineering have been presented.

The treatment of fractional order calculus in this paper is suggestive rather than rigorous in order to capture the reader's interest while simultaneously offering a hint of its potential as a research tool. A few applications have begun to appear, but they are still in the incipient stage of development.

This article presented several case studies involving the implementation of FOC-based models, whose results demonstrate the importance of Fractional Order Calculus. We strongly hope they will serve as motivation for the development of new applications.

\section{Referências}

[1] B.M. Hambly and M.L. Lapidus, Trans. Amer. Math. Soc. 358, 28 (2006).

[2] F. Mainardi, Chaos, Solitons \& Fractals 7, 1461 (1996).

[3] A.L. Méhauté, New Journal of Chemistry 14, 207 (1990).

[4] K.B. Oldham and J. Spanier, The Fractional Calculus: Theory and Applications of Differentiation and Integration to Arbitrary Order (Dover Publication, Mineola, 2006).

[5] R.L. Bagley and P.J. Torvik, Journal of Rheology 3, 133 (1986).

[6] J.A.T. Machado, Journal Systems Analysis, Modelling, Simulation 27, 107 (1997).

[7] J.A.T. Machado and A. Azenha, in: IEEE Int. Workshop on Advanced Motion Control, Coimbra, p. 548-553 (1998).

[8] J.A.T. Machado, Nonlinear Dynamics, Chaos, Control and Their Applications to Engineering Sciences 4, 99 (2002).

[9] H.M. Ozaktas, O. Arikan, M.A. Kutay and G. Bozdagi, IEEE Trans. on signal Processing 44, 2141 (1996).

[10] A. Oustaloup, IEEE Trans. on Cicuits and Systems 28, 1007 (1981).

[11] B. Mathieu, L.L. Lay and A. Oustaloup, in: IEEESMC/IMACS Symposium on Control, Optimization and Supervision, Lille, p. 952-956 (1996).
[12] B. Ross, Mathematics Magazine 50, 115 (1977).

[13] M.D. Ortigueira, J.A.T. Machado and J.S. da Costa, IEEE Proceeedings: Vision, Image and Signal Processing 152, 846 (2005).

[14] O.P. Agrawall, Nonlinear Dynamics 29, 145 (2002).

[15] L. Ma and G.V. Barbosa-Cánovas, Journal of Texture Studies 27, 307 (1996).

[16] R.C.L. Bosworth, Nature 137, 447 (1946).

[17] N.M.F. Ferreira, J.A.T. Machado, A.M.S.F. Galhano and J.B. Cunha, in: Proceedings of the $2^{\text {nd }}$ IFAC Workshop on Fractional Differentiation and its Applications,p. 374-379, v. 2 (2006).

[18] M.F.M. Lima, J.A.T. Machado and M. Cris, in: Proceedings of the $2^{\text {nd }}$ IFAC Workshop on Fractional Differentiation and its Applications, p. 12-18, v. 2 (2006).

[19] C.A. Monje, F. Ramos, V. Feliu and B.M. Vinagre, in: IET Control Theory and Applications, v. 2 (2007).

[20] M.F. Silva and J.A.T. Machado, Journal of Vibration and Control 12, 1483 (2006).

[21] B.M. Hambly, S. Howison and T. Kluge, Quantitative Finance 9, 937 (2009).

[22] S.A. David and E.L. Ursini, in: 6th Brazilian Conference on Dynamics, Control and Their Applications, São Jose do Rio Preto, v. 1, p. 999-1003 (2007).

[23] M.B. Garman and M.J. Klass, Journal of Business 53, 67 (1980).

[24] W.T. Shaw, Modelling Financial Derivatives with Mathematica (Cambridge University Press, Cambridge, 1998).

[25] M.B. Garman, Descomposición de los Elementos Componentes del VeR Dentro de una Cartera de Inversión (Financial Engineering Associates, Inc., Berkeley, 1997), p. 2-7.

[26] L. Akdeniz and W.D. Dechert, in: Proc: Second International Conference on Computing in Economics and Finance, Geneva (1996).

[27] E.W. Anderson and L.P. Hansen, in: Second International Conference on Computing in Economics and Finance, Geneva, p. 1-13 (1996).

[28] E. Scalas, R. Gorenflo and F. Mainardi, Physica A 284, 376 (2000).

[29] S.A. David, in: Proceddings of the 2007 Iternational Conference on Computational and Mathematical Methods in Science and Engineering, Chicago, p. 148-158, v. 1 (2007) 